\title{
Dynamic Modelling of an Automated Vehicle Storage and Retrieval System and a Simulation Analysis of its Efficiency
}

\author{
Zou Xia(1,2), Wu Yaohua(3), Xia Delong(3),Chen Yunxia(3) \\ (1) School of Management Science and Engineering, Shandong University of Finance and Economics, Jinan, CHINA \\ (2) School of Management, Shandong University, Jinan, CHINA \\ (3) School of Control Science and Engineering, Shandong University, Jinan, CHINA
}

\section{SUMMARY}

In this paper operating-time models for single and multiple instructions are set up considering an AVS/RS (automated vehicle storage and retrieval system). The operation times of AVS/RS and $A S / R S$ (automated storage and retrieval system) are simulated in different situations by changing the shelf structure and order density. The results show that the AVS/RS is more efficient than the $A S / R S$ in all situations. Furthermore, the numbers of rows and columns of storage shelves greatly influence the operation time. The graph of operation-time compression ratio against number of columns shows an inverted U-type distribution, and the compression ratio decreases and ultimately tends to zero as the number of rows is increased. Also, the order density affects the efficiency difference between the two systems: the higher the order density, the higher the AVS/RS operatingtime compression rate. Finally, compared with the AS/RS, the AVS/RS operating-time compression ratio improves greatly with increasing density and number of rows because of parallel operations, whereas with decreasing density and number of rows the AVS/RS advantages are gradually lost and the compression ratio decreases and eventually even reaches zero.

KEY WORDS: $A V S / R S$, operating-time modelling, storage shelves structure, order density.

\section{INTRODUCTION}

A traditional automated storage and retrieval system (AS/RS) comprises a high-rise rack, automatic stackers, and a warehouse management system (WMS). It can achieve online control of equipment through the WMS, carry out serial of operations, and is characterized by the full use of storage space, low labour costs, and improved storage operation rate [1]. An autonomous vehicle storage and retrieval system (AVS/RS) [2] is a new AS/RS-based technology that achieves intelligent three-dimensional storage. The AVS/RS replaces the AS/RS automatic stacker with shuttle cars, track, and hoist. Controlled by the WMS and other management systems, parallel operations are conducted to improve further the operation efficiency. Given 
that an AVS/RS and an AS/RS have different characteristics, the present study seeks to determine which would be more efficient for a specific set of operation needs.

\section{LITERATURE REVIEW}

Regarding operation strategy and algorithm optimization, Fang and Tang [7] and Zhang et al. [8] proposed an artificial wolf-pack algorithm to improve AVS/RS warehouse operation efficiency. Based on the allocation and product-layout model for class-based storage, Roshan et al. [9] proposed a multi-objective optimization model that uses energy-consumption optimization and energy sustainability to allocate items to locations while prioritizing minimum AS/RS movement. Wauters et al. [10] studied a mini-load AS/RS system with a dual-shuttle crane and introduced different heuristic assignment strategies. Gharehgozli et al. [11] studied the total travel time of cranes in a container-terminal AS/RS system and validated the two-phase solution to the optimal solution by establishing a continuous-time integer-programming model. However, although the above-mentioned studies are relevant here, their research objectives differ from those presented here.

Regarding system-performance analysis, Marchet et al. [12] proposed an analytical model for estimating product motion performance. For container freight, Vis [13] analyzed and compared the performance of a manned carrier and an automatic stacker. For traditional manufacturing, Fonseca et al. [14] calculated a score for each conveyor type by weighted assessment methods and the expected value of risk decisions, selecting conveyors based on the suitability score and validating the accuracy of the applicability score through factory data. Wu [15] studied a threedimensional simulation platform for an automatic car access system, designed various ways to allow this platform to obtain the route sequence of cars, and exhibit and verify the results of the dynamic model in three-dimensional animation. Zhao et al. [16] constructed a simulation model for a patrol-type automatic trolley access system, using orthogonal experimentation to simulate the speed, acceleration, and assignment of goods locations; they showed that the speed affected the system quite differently depending on the number of columns in the warehouse. Dhiyaeddine Metahri [17] proposed an analytical model for the expected retrieval-travel time for a free-fall flow rack under a dedicated storage assignment policy to optimize the dimensions of the rack, assess the system throughput, and evaluate different storage policies. The aforementioned studies have some relevance to this operating-time modelling, but the difference is that this one aims at modelling the AVS/RS operation time. Also, by adjusting the shelf structure and the order density, we analyze tendencies in AVS/RS and AS/RS operation times as well as differences between the two systems.

The above discussion shows that AVS/RS research is relatively poor and has focused mainly on scheduling control and strategy optimization. There is no research to compare AVS/RS and AS/RS performance from the point of different shelf structures and order densities. For this reason there is merit of the present research. 


\section{PROBLEM DESCRIPTION}

AVS/RS and AS/RS differ fundamentally in their operation models, since an AVS/RS involves parallel operations. In an AVS/RS, the combination of shuttles and an elevator replaces the stacker in an AS/RS. The shuttles are used to implement horizontal movement and the elevator is used to implement vertical movement in a single laneway, as shown in Figure 1.

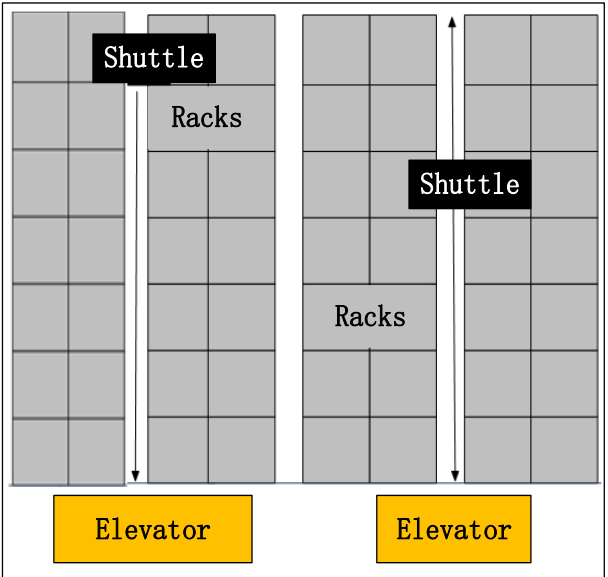

Top View

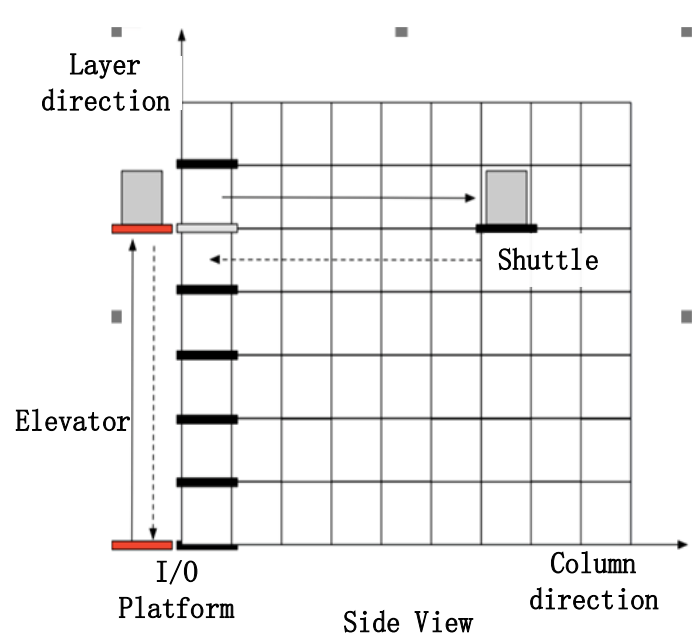

Side View

Fig. 1 Top view (left) and side view (right) of an automated vehicle storage and retrieval system (AVS/RS)

However, an AVS/RS requires many devices, not only making it more expensive but also complicating scheduling and management of those devices $[18,19]$. Selecting an efficient and applicable automated warehousing system requires scientific decisions to be made. The size of the warehouse, the structure of the shelves, the number of items, and the density of orders are all factors to be considered. Here, as an example, we take an AVS/RS system and analyze its operating principles. We establish a system operation-time model aiming to quick response to instructions, and we analyze how the system efficiency changes when the shelf structure and order density are varied. The simulation results will help logistics enterprises to plan reasonable logistics systems and improve their operation efficiency.

\section{MODELLING AVS/RS OPERATION TIME}

An AVS/RS operates in parallel, complicating in that way any model of its operation time. In this research, an AVS/RS is used as an example in order to model the operation time according to the characteristics of the system operation. The method for modelling the AS/RS operation time is the same as that for an AVS/RS [20], meaning it is not fully elaborated.

\subsection{EQUIPMENT ACTION ANALYSIS}

The operating process of devices in warehousing operations can be divided into many sub processes, such as identification, forking, handling, unloading, and returning in a specific order. Thus, the timing of each operation and the motion of the shuttles and elevator should be analyzed to establish a model for the system operation time; see Table 1 for details. 
Table 1 Operation actions for AVS/RS equipment

\begin{tabular}{|c|c|c|c|c|}
\hline $\begin{array}{l}\text { Completion } \\
\text { time }\end{array}$ & $\begin{array}{l}\text { Operation } \\
\text { equipment }\end{array}$ & Task & $\begin{array}{l}\text { Completion } \\
\text { time }\end{array}$ & $\begin{array}{l}\text { Action } \\
\text { composition }\end{array}$ \\
\hline \multirow{6}{*}{ OTS } & \multirow{6}{*}{ Shuttle } & Machine movement (no load) & $t_{11}$ & $\begin{array}{l}\text { Horizontal } \\
\text { movement }\end{array}$ \\
\hline & & $\begin{array}{l}\text { Identification goods or goods } \\
\text { location }\end{array}$ & $t_{12}$ & Identification \\
\hline & & Fork the goods & $t_{13}$ & $\begin{array}{l}\text { Reaching } \\
\text { Lifting }\end{array}$ \\
\hline & & Machine movement (load) & $t_{14}$ & $\begin{array}{l}\text { Horizontal } \\
\text { movement }\end{array}$ \\
\hline & & Unloading & $t_{15}$ & $\begin{array}{l}\text { Fork drop } \\
\text { Fork recovery }\end{array}$ \\
\hline & & Waiting & $t_{16}$ & Waiting instruct \\
\hline \multirow{6}{*}{ OTE } & \multirow{6}{*}{ Elevator } & Machine movement (no load) & $t_{21}$ & Vertical movement \\
\hline & & $\begin{array}{l}\text { Identification goods or goods } \\
\text { location }\end{array}$ & $t_{22}$ & Identification \\
\hline & & Fork the goods & $t_{23}$ & $\begin{array}{l}\text { Reaching } \\
\text { Lifting }\end{array}$ \\
\hline & & Machine movement (load) & $t_{24}$ & Vertical movement \\
\hline & & Unloading & $t_{25}$ & $\begin{array}{l}\text { Fork drop } \\
\text { Fork recovery }\end{array}$ \\
\hline & & Waiting & $t_{26}$ & Waiting instruct \\
\hline
\end{tabular}

OTS: the time of shuttle to complete one instruction

OTE: the time of elevator to complete one instruction

\subsection{EQUIPMENT ACTION ANALYSIS}

According to Table 1, the following Eqs. can be obtained as Eqs. (1) and (2):

$$
\begin{aligned}
& \text { OTS }_{i}=t_{i 11}+t_{i 12}+t_{i 13}+t_{i 14}+t_{i 15}+t_{i 16} \\
& \text { OTE }_{i}=t_{i 21}+t_{i 22}+t_{i 23}+t_{i 24}+t_{i 25}+t_{i 26}
\end{aligned}
$$

where the parameters meet the following assumptions.

1) Under ideal conditions, the operation times $t_{i 12}$ and $t_{i 22}$ are negligibly short.

2) Protruding and retracting fork consume the same, fixed times which are defined as $t_{s s}$ and $t_{t l}$ for the time of fork lifting and putting, respectively.

3) The shuttles and elevator stay still after finishing an operation instruction until they receive the next one.

4) The waiting time of the stacker is zero, namely $t_{i 16}=t_{i 26}=0$.

Eqs. (1) and (2) can be simplified as below.

$$
\begin{aligned}
& O T S_{i}=t_{i 11}+t_{i 14}+4 t_{s s}+2 t_{t l} \\
& \text { OTE }_{i}=t_{i 21}+t_{i 24}+4 t_{s s}+2 t_{t l}
\end{aligned}
$$


According to Eqs. (3) and (4), OTS $S_{i}$ depends on $t_{i 11}$ and $t_{i 14}$. When calculating $t_{i 11}$, the distance travelled by the device is determined by its location and those of the goods. The former instruction determines the device location whereas the latter instruction determines the goods locations. When calculating $t_{i 14}$, the locations and destinations of the goods determine the distances travelled by the device, which are determined in turn by the current order. The time required by the device to travel (including $t_{i 11}, t_{i 14}, t_{i 21}$, and $t_{i 24}$ ) is calculated according to Eq. (5), which considers the relationship among travel time and distance, device acceleration, and speed.

$$
r t=\left\{\begin{array}{l}
2 \sqrt{\frac{l}{a}}, l<\frac{v^{2}}{a} \\
\frac{l}{v}+\frac{v}{a}, l \geq \frac{v^{2}}{a}
\end{array}\right.
$$

$r t$ is running time of equipment, $l$ travelling distance, $v$ running speed of equipment and $a$ is running acceleration of equipment.

\subsection{MODELLING OF MULTI-INSTRUCTION OPERATION TIME}

Since the AVS/RS devices operate in parallel and the operation is more complex under multioperation instructions, we use the output timing diagram to show the relationship among equipment operations, as shown in Figure 2.

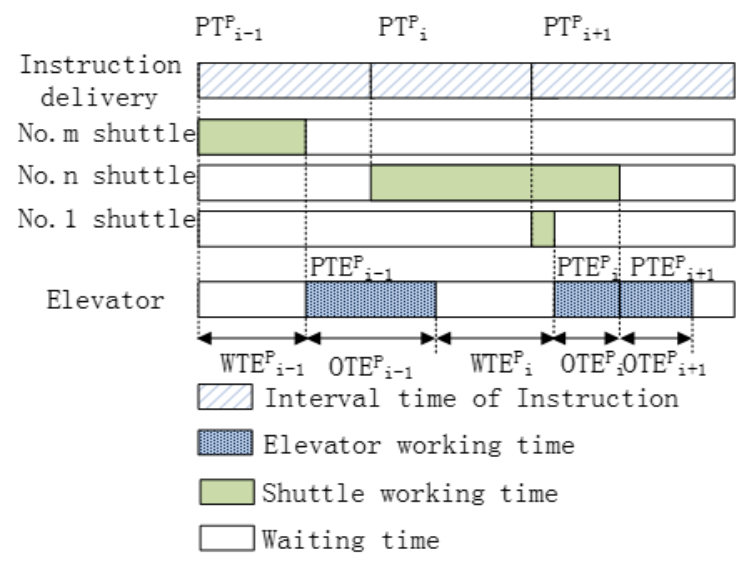

Fig. 2 Time sequence diagram for output operation of AVS/RS

Figure 2 shows that multiple shuttles can move simultaneously to complete horizontal operation, but only single elevator completes vertical movement. The operation time of AVS/RS driven by the random orders can be converted into the sum of the instruction-execution time and the elevator waiting time. The model is expressed as below.

$$
\operatorname{Total}_{N}^{S}=\sum_{i=1}^{N} O T E_{i}+\sum_{i=1}^{N} W T E_{i}
$$

Where $W T E_{i}$ is determined by the final and release times of vertical instruction $i$. If the order issuing time is $P T_{i}$ and the vertical-instruction issuing time is, $P T_{i}$ then Eq. (6) is converted to Eq. (7): 


$$
\operatorname{Total} T_{i}^{S}=\operatorname{Max}\left(\operatorname{Total} T_{i-1}^{S}, P T E_{i}\right)+O T E_{1}
$$

The values of $O T E_{i}$ and $P T E_{i}$ must be calculated. Eq. (5) shows how to calculate $O T E_{i}$. However, it is more complicated to calculate the vertical-instruction issuing time. We do so using the following algorithm.

- Step 1: Construct a dynamic instruction set for each device, including the elevator and shuttles. Take the order of the total instruction set as $K$, and then sort the instructions in accordance with the release time from small to large. Take the dynamic operation instruction sets of the shuttle bus and the elevator as $K \frac{s}{h}(h=1 \ldots H)$ and $K^{e}$, respectively.

In the initial state $K, K \frac{S}{h}$ and $K^{e}$ as empty set, $K$ continues to accept the new operation instructions.

- $\quad$ Step 2: Repeat 2.1 and 2.3 until $K$ is empty set.

- Step 2.1: When the system receives a operation instruction, if $P_{i}=1$ then go to 2.2; if $P_{i}=-1$ then go to 2.3 .

- Step 2.2: Delete this instruction from $K$ and put it into $K^{e}$; furthermore, let $P T E_{i}=P T_{i}$. Sort instructions based on $P T E_{i}$ from small to large, waiting for the elevator to execute the order. Then the elevator executes the operation instructions in turn, and $\operatorname{Total}_{i}=\operatorname{Max}\left(\operatorname{Total}_{i}, P T E_{i}\right)+O T E_{i}$. Go to Step 2.4.

- $\quad$ Step 2.3: Delete this instruction from $K, \operatorname{read} Y_{i}$ from $L_{i}$ and put it into $K \frac{S}{h}\left(h=1 \ldots y_{i}\right)$, set up $P T S_{i}^{h}=P T_{i}$. Sort instructions based on $P T E_{i}$ from small to large, waiting for the elevator to execute the order. Then let $\operatorname{TotalTS}_{i}=\operatorname{Max}\left(\operatorname{TotalTS}_{i-1}^{h}, P T S_{i}^{h}\right)+O T S_{i}^{h}$. Go to Step 2.5 . $^{2}$

- Step 2.4: Delete this instruction from $K$, read $Y_{i}$ from $L_{i}$ and put it into $K \frac{s}{h}\left(h=y_{i}\right)$. Sort instructions based on $\mathrm{PTH}_{i}$ from small to large and execute the order in turn, and $\operatorname{Total}_{i}=\operatorname{Max}\left(\operatorname{TotalT}_{i}, P T H_{i}\right)+O T H_{i}$. Delete the instruction.

- $\quad$ Step 2.5: Delete this instruction from $K$, put it into $K^{e}$, and make PTE $E_{i}=$ TotalTS $_{i}^{h}$. Sort instructions based on $P T E_{i}$ from small to large and execute the $K \frac{S}{h}\left(h=y_{i}\right)$ order in turn.

\section{EMPIRICAL ANALYSIS}

Since the AVS/RS and AS/RS are frequently used in business-to-consumer logistics centres we take them as an example two systems are simulated and their properties are assessed. A miniload stacker is adopted to represent the AS/RS, whose horizontal and vertical velocities are both $4 \mathrm{~m} / \mathrm{s}$ and whose acceleration is $2 \mathrm{~m} / \mathrm{s}^{2}$. For the AVS/RS, the vertical velocity of the elevator is 4 $\mathrm{m} / \mathrm{s}$, the acceleration is $2 \mathrm{~m} / \mathrm{s}^{2}$, the horizontal velocity of a shuttle is $4 \mathrm{~m} / \mathrm{s}$, and the acceleration is $2 \mathrm{~m} / \mathrm{s}^{2}$. These three devices share the same loading/unloading operation time unit of $4 \mathrm{~s}$, which includes fork-out, fork-back, and fork-up/fork-down. 
Because the shelf parameters and order structure affect the efficiency of each system, we divide the simulation into three different environments.

- Environment 1: Assuming that orders arrive at once and the shelf structure changes, the efficiencies of AVS/RS and AS/RS are simulated. We regard each system as a first come, first served (FCFS) queuing system and calculate the output.

- Environment 2: Assuming that the shelf structure is fixed but the orders arrival time is changed, the efficiency of two systems is simulated. Let the order-arrival time intervals which are independent of each other and have the same parameters follow a negative exponential distribution. Additionally, let the shelf consists of 10 layers and 100 columns, and adjust the order density so that the order time interval follows an index distribution whose mean value ranges from zero to 100 . The two systems are designed to complete 100 simulations of 200 orders.

- Environment 3: Change the shelf shape and order arrival time as well, in order to assess how these two factors affect each system. The number of shelf layers is $L=10$, the number of columns starts at 10 and is then increased to 100 , and the order-arrival time interval starts at zero and is then increased to $100 \mathrm{~s}$. The two systems undergo 100 simulation tests for 200 orders.

\section{RESULTS AND DISCUSSION}

\subsection{EFFECT OF SHELF STRUCTURE ON SYSTEM PERFORMANCE}

\subsubsection{EFFECT OF COLUMN NUMBER ON SYSTEM EFFICIENCY}

We adjust the number of shelf columns $C$, ranging from 10 to 100 with the number of shelf layers fixed at $L=10$. The simulation results of the two systems are analyzed statistically and the task completion time of each system under different circumstances is obtained, as given in Table 2.

Table 2 Simulation results for time compression rate

\begin{tabular}{|c|c|c|c|c|}
\hline $\begin{array}{c}\text { Number of columns } \\
C\end{array}$ & $\begin{array}{c}\text { Task completion time } \\
\text { of } A S / R S \\
\text { Total }^{S}\end{array}$ & $\begin{array}{c}\text { Task completion time } \\
\text { of AVS } / R S \\
{\text { Total } T^{p}}^{p}\end{array}$ & 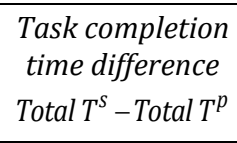 & $\begin{array}{c}\text { Time compression rate \% } \\
\frac{{\text { Total } T^{s}-\text { Total }^{p}}^{\text {Total }^{s}}}{}\end{array}$ \\
\hline 10 & $2,768.54$ & $2,367 \cdot 30$ & 401.24 & 14. 49 \\
\hline 20 & 3, 559. 22 & $2,418.16$ & $1,141.06$ & 32.06 \\
\hline 30 & $4,587.78$ & $2,418.50$ & $2,169.28$ & 47.28 \\
\hline 40 & $5,531.58$ & $2,449.70$ & $3,081.88$ & 55.71 \\
\hline 50 & $6,772.62$ & $2,460.58$ & $4,312.04$ & 63.67 \\
\hline 60 & $7,408.60$ & $2,485.40$ & 4, 923.2 & 66.45 \\
\hline 70 & $8,660.02$ & $2,570.86$ & $6,089.16$ & 70.31 \\
\hline 80 & $9,665.76$ & $2,601.44$ & $7,064.32$ & 73.07 \\
\hline 90 & $10,663.88$ & $2,557.40$ & $8,106.48$ & 76.02 \\
\hline 100 & $11,633.78$ & $2,633.84$ & 8, 999. 94 & 77.36 \\
\hline
\end{tabular}

Total $T^{s}$ : AVS/RS operating time

Total $T^{p}$ : AS/RS operating time 
The trend and curve suitable for the system efficiency by the number of shelf columns are obtained from Table 2 and shown in Figure 3.

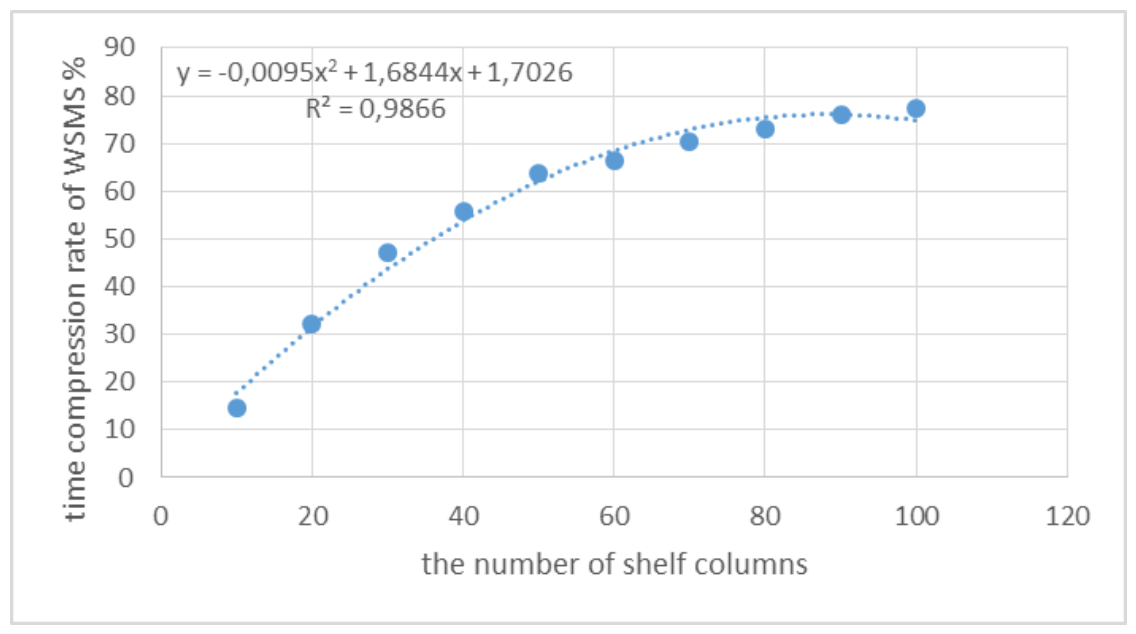

Fig. 3 Simulation results for time compression rate against number of shelf columns

Regarding the system efficiency, we draw the following conclusions from the simulation data.

1) When orders arrive all at once and the number of shelf layers is constant at $L=10$ and the number of shelf columns ranges from 10 to 100 . The results demonstrate that the AVS/RS operation time is less than the AS/RS one.

2) When the number of shelf layers is 10 , the time compression ratio increases with the number of shelf columns.

\subsubsection{EFFECT OF COLUMN NUMBER ON ORDER WAITING TIME}

The system timeliness is reflected by the order waiting time. The simulation resulting for the order waiting time of each system are shown in Figure 4.

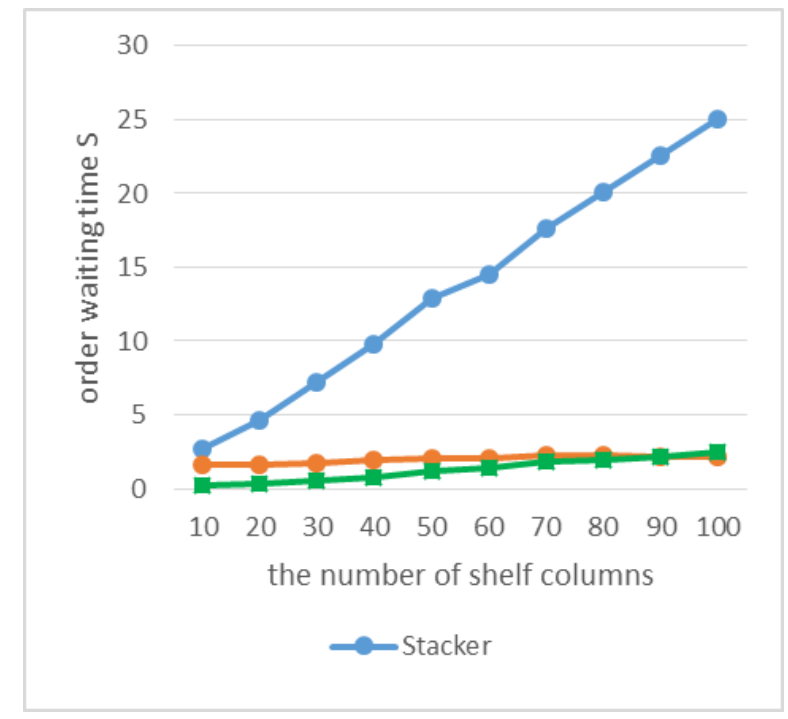

Fig. 4 Simulation results for order waiting time against number of shelf columns

Regarding the task waiting time of equipment, we draw the following conclusions from the simulation data: 
1) When orders arrive all at once and the number of shelf layers is constant at $L=10$ and the number of shelf columns ranges from 10 to 100 . The results show that the AS/RS task waiting time is longer than the AVS/RS one.

2) When the number of shelf layers is 10 and the number of shelf columns ranges from 10 to 100 , the AVS/RS task waiting time increases, obviously indicating that the stacker is busy. In the AVS/RS, the waiting time for the shuttles is lower and increases slightly, while the waiting time for the elevator is higher and increases faster..

3) When the number of shelf layers is 10 and the number of shelf columns ranges from 10 to 100 , the task of the stacker has to pause longer, and the pausing time for the elevator and the shuttle increases slowly.

\subsubsection{EFFECT OF COLUMN NUMBER ON SYSTEM EFFICIENCY}

When the shelves columns are same, in different shelf layers $L$ ( $L$ increased from 10 to 100 ), they simulate the completion time for the two systems under different conditions. Particular case is as shown in Table 3. The time compression ratio by number of shelf layers is shown in Figure 5.

Table 3 Simulation results for time compression ratio

\begin{tabular}{|c|c|c|c|c|}
\hline & $A S / R S$ & $A V S / R S$ & Comparison & and analysis \\
\hline Number of layers & $\begin{array}{c}\text { Task completion time } \\
\text { Total } T^{s}\end{array}$ & $\begin{array}{c}\text { Task completion } \\
\text { time } \\
\text { Total }^{p}\end{array}$ & 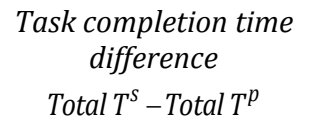 & $\begin{array}{c}\text { Time compression rate } \\
\frac{{\text { Total } T^{S}-\text { Total }^{p}}^{\text {Total } T^{S}}}{}\end{array}$ \\
\hline 10 & $2,768.54$ & $2,416.02$ & 352.52 & 12. 73 \\
\hline 20 & $3,548.90$ & $3,154.82$ & 394.08 & 11. 10 \\
\hline 30 & $4,646.04$ & $4,180.54$ & 465.50 & 10.01 \\
\hline 40 & $5,601.28$ & $5,197.82$ & 403. 46 & 7. 20 \\
\hline 50 & $6,536.38$ & $6,316.84$ & 219.54 & 3. 36 \\
\hline 60 & $7,294.30$ & $7,194.86$ & 99.44 & 1. 36 \\
\hline 70 & $8,216.72$ & $8,165.36$ & 51.36 & 0.63 \\
\hline 80 & $9,353.28$ & $9,311.44$ & 41.84 & 0.45 \\
\hline 90 & $10,633.12$ & $10,570.14$ & 62.98 & 0.59 \\
\hline 100 & $11,727.04$ & $11,605.46$ & 121.58 & 1. 04 \\
\hline
\end{tabular}

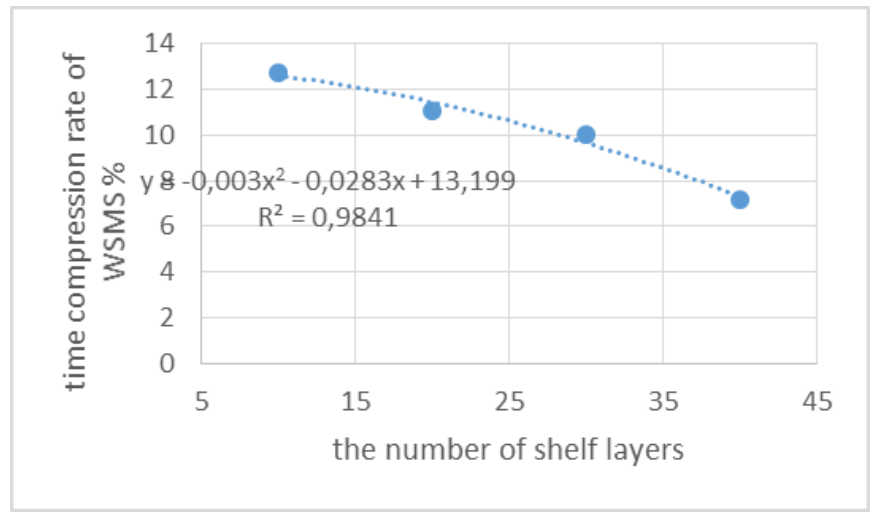

Fig. 5 Simulation results for time compression ratio against number of shelf layers 
Analyzing the system efficiency from the simulation data, we draw the following preliminary conclusions.

1) When the orders arrive simultaneously and instantaneously, the number of shelf columns is unchanged $(C=10)$ and the number of shelf layers $L$ is increased from 10 to 100 , the AVS/RS task completion time is less than the AS/RS one.

2) When the number of shelf columns is constant $(C=10)$ and the number of shelf layers $L$ increases from 10 to 100 , the AVS/RS-to-AS/RS time compression ratio decreases. In other words, the difference in efficiency between the two systems decreases.

\subsubsection{EFFECT OF LAYER NUMBER ON ORDER WAITING TIME}

To assess the timeliness of each system, we obtain the average pausing time of each system from the simulation data, as shown in Figure 6. Regarding task waiting time of the equipment, we draw the following preliminary conclusions.

1) In the case the orders arrive simultaneously and instantaneously, and with a fixed number of shelf layers $(L=10)$ and the number of shelf columns increasing from 10 to 100 , the AVS/RS shuttle can complete the task immediately without pausing, and task pausing time for elevator increases significantly, as the elevator is busy and it become a bottleneck of the system. Meanwhile, the AS/RS task pausing time increases slowly at a rate that is much lower than that in the AVS/RS.

2) With more shelf layers, the AS/RS is more efficient than the AVS/RS.

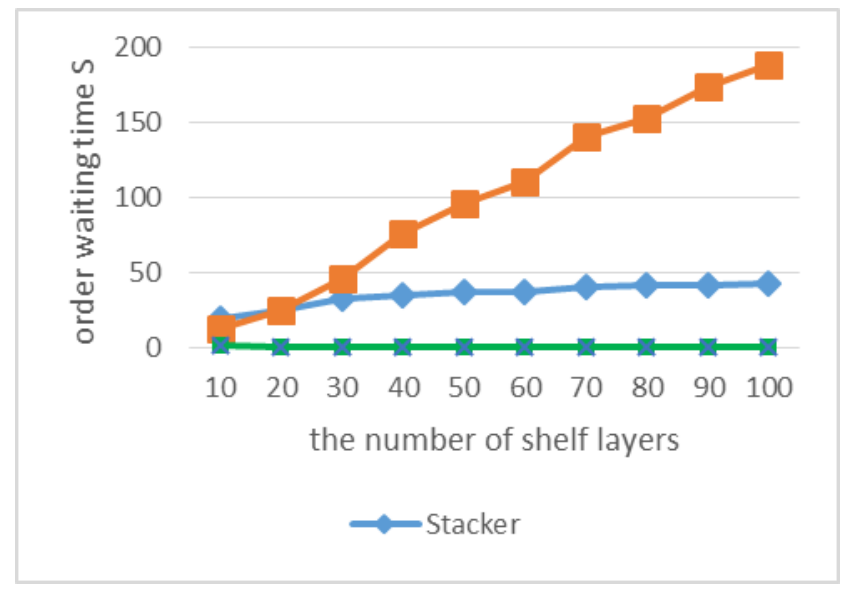

Fig. 6 Simulation results for task waiting time against number of shelf layers

\subsection{EFFECT OF ORDER DENSITY ON SYSTEM PERFORMANCE}

\subsubsection{EFFECT OF ORDER DENSITY ON SYSTEM EFFICIENCY}

We set up that the shelf contains 10 layers and 100 columns, adjust the density of orders with the arrival time interval following the index distribution of the mean of 0 and 100 in turn. The time difference between the two systems completing 200 random orders is simulated, and simulation is repeated 100 times. The simulation results in Figure 7 show how the operation 
time of each system varies with the order density, which helps us assess the operation efficiency of each system.

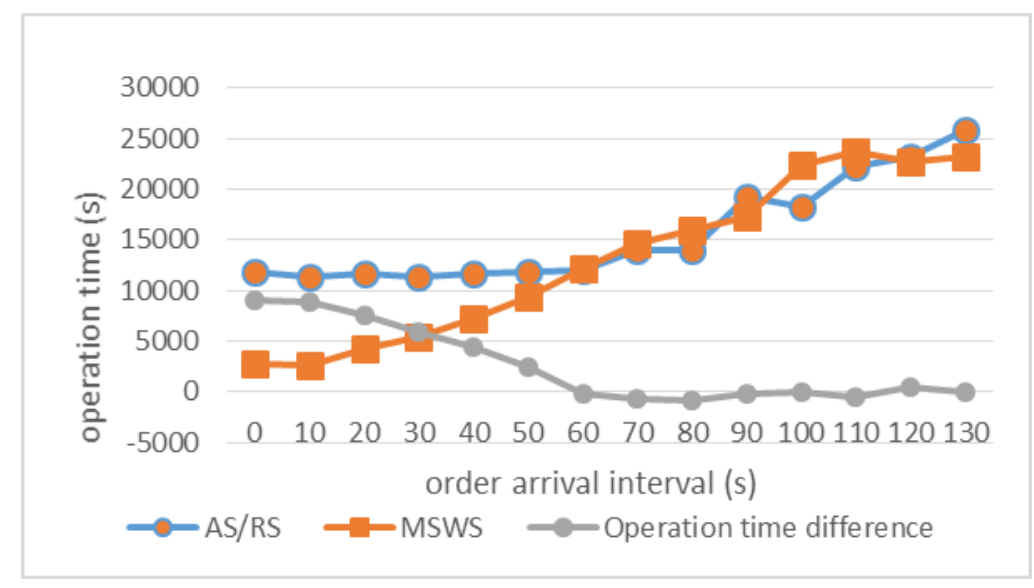

Fig. 7 Simulation results for system operation time against order arrival interval

From the simulation results that when the mean order interval is zero, the AS/RS task completion time is $11,844.6 \mathrm{~s}$ and the AVS/RS task completion time is $2,746.5 \mathrm{~s}$. As the mean order interval increases, the operation completion time of each system increases, the AVS/RS increases slower than AS/RS. When the mean order interval is $60 \mathrm{~s}$, the completion times of the two systems are approximately equal. When the mean order interval is $70-120 \mathrm{~s}$, the AVS/RS completion time is longer than the AS/RS one.

\subsubsection{EFFECT OF ORDER DENSITY ON EQUIPMENT UTILIZATION}

From the simulation results as shown in figure 8 that when the mean order time is zero, the AS/RS stacker utilization rate is $56.8 \%$, the AVS/RS elevator utilization rate is $75.8 \%$, and the average shuttle utilization rate is $23.9 \%$. Increasing the time between orders, the equipment utilization ratio of each system decreases and the AVS/RS elevator and shuttle efficiencies decrease significantly. For a mean order interval of $30 \mathrm{~s}$, the elevator efficiency is less than the stacker one. When order interval time is above $30 \mathrm{~s}$, the AVS/RS equipment utilization rate is close to the fixed value of $2 \%$ and the AS/RS equipment utilization rate is close to $30 \%$.

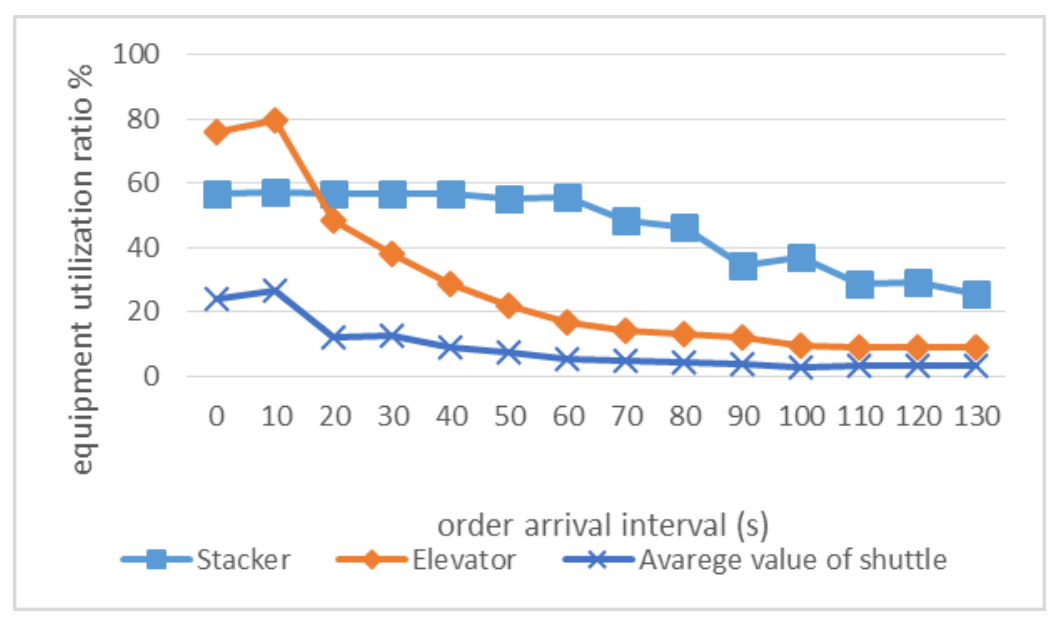

Fig. 8 Simulation results for equipment utilization against order interval time 
The overall AVS/RS equipment utilization rate is determined mainly by the elevator utilization. As the system efficiency is concerned, when the order density is greater, the AVS/RS equipment utilization rate is higher. With decreasing order density, the equipment utilization rate of the AS/RS and the AVS/RS are both high, and the shuttle utilization rate is extremely low in particular.

\subsection{EFFECT OF DUAL-FACTORS ON SYSTEM PERFORMANCE}

We conduct simulations to determine how the efficiency of each system changes when the order density and the shelf structure are changed simultaneously. Because civil construction is highly restricted, 10 layers shelve is used and the number of shelf columns in the AVS/RS is adjusted to match the different order density. Figure 9 shows how varying the number of shelf columns and the order density affects the time difference between the two systems.

For the simulation results, the larger the order density and the greater the number of shelf columns, the more evident the efficiency of the parallel AVS/RS operation is. With lower order density and fewer shelf columns, the advantages of the parallel efficiency of the AVS/RS are gradually reduced, and AVS/RS efficiency is affected by order density and the number of shelf columns.

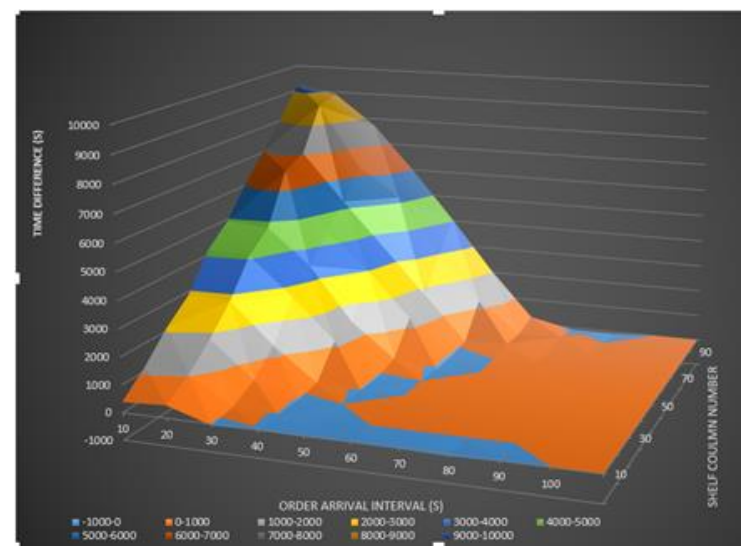

Fig.9 Variation of time difference with order density and number of shelf columns

\section{CONCLUSIONS}

A model of AVS/RS operation time is established and the difference between an AVS/RS and an AS/RS is compared when the shelf structure and order density are changed. The conclusions are as follows:

1) When the number of shelf layers is fixed and the number of shelf columns ranges from 10 to 100 , the AVS/RS is obviously more advantageous to the AS/RS and saves more time. The time compression rate includes an inverted U-type distribution because the shuttles perform parallel operations in the AVS/RS, reducing the time of horizontal movement.

2) When the number of shelf columns is fixed and the number of shelf layers ranges from 10 to 100 , the AVS/RS shows no advantages over the AS/RS. With more layers, only one lift is responsible for vertical movement in the AVS/RS so it becomes the bottleneck of the system. 
3) The greater the order density, the more obvious the benefits of the AVS/RS are. With decreasing order density, the two systems tend to have consistent efficiency.

4) The greater the order density and the larger the number of shelf columns, the more obvious the AVS/RS parallel-operation efficiency is. With lower order density and fewer shelf columns, the parallel benefit is reduced and the elevator efficiency determines that of the AVS/RS system.

Because of time limitation, shelf change is limited to a certain range. In addition, the normal distribution of orders is assumed. We hope that the reliability of the conclusion will be further verified with real orders in the future.

\section{REFERENCES}

[1] K.J. Roodbergen and I. F. A. Vis, A survey of literature on automated storage and retrieval systems, European Journal of Operational Research, Vol. 194, No. 2, pp. 343-462, 2009. https://doi.org/10.1016/j.ejor.2008.01.038

[2] Y.J. Fang and M. Tang, AVS/RS system conflict and deadlock control strategy, Automation and Instrumentation, Vol. 29, No. 12, pp. 1-4, 2014.

http://doi.org/10.19557/j.cnki.1001-9944.2014.12.001

[3] Y. M. Wu, Y.Q. Liu, D. Zhang, H. Zhao, Research on dynamic scheduling of RGV based on genetic algorithm, Lifting and Transport Machinery, vol. 2, No.6, pp. 20-23, 2012. https://doi.org/10.3969/j.issn.1001-0785.2012.06.006

[4] B.Y. Ekren, S.S. Heragu, A. Krishnamurthy and C.J. Malmborg, An approximate solution for semi-open queueing network model of an autonomous vehicle storage and retrieval system, Transactions on Automation Science and Engineering, Vol. 10, No. 1, pp. 205-215, 2013. https://doi.org/10.1080/00207543.2011.639815

[5] B.Y. Ekren, S.S. Heragu, A. Krishnamurthy and C.J. Malmborg, Simulation based experimental design to identify factors affecting performance of AVS/RS, Computers \& Industrial Engineering, Vol. 58, No. 1, 2009.

http://doi.org/10.1016/j.cie.2009.10.004

[6] H.Y.K. Lau and Y. Zhao, Joint scheduling of material handling equipment in automated air cargo terminals, Computers in Industry, Vol. 57, No. 5, pp. 398-411, 2006. https://doi.org/10.1016/j.compind.2005.11.003

[7] Y.J. Fang and M. Tang, AVS/RS system dynamics analysis and decision model, Automation Instrumentation, Vol. 36, No. 1, pp. 18-22, 2015. http://doi.org/10.16086/j.cnki.issn1000-0380.201501005

[8] S.J. Zhang, Y.J. Fang, Y. He and Y. Xiao, Optimization of AVS/RS multi-batch container storage location based on simulated annealing algorithm, Journal of Wuhan University (Engineering Science), Vol. 49, No. 2, pp. 315-320, 2016. https://doi.org/10.14188/j.1671-8844.2016-02-027

[9] K. Roshan, A. Shojaie and M. Javadi, Advanced allocation policy in class-based storage to improve AS/RS efficiency toward green manufacturing, International Journal of Environmental Science and Technology, Vol. 6, No. 1, pp. 1-12, 2018. https://doi.org/10.1007/s13762-018-1921-6 
[10] T. Wauters, F. Villa, J. Christiaens, R. A. Valdesc, G. V. Berghea, A decomposition approach to dual shuttle automated storage and retrieval systems, Computers \& Industrial Engineering, Vol. 101, pp. 325-337, 2016. https://doi.org/10.1016/j.cie.2016.09.013

[11] A.H. Gharehgozli, Y. Yu and R.D. Koster, An exact method for scheduling a yard crane, European Journal of Operational Research, Vol.235, No.2, pp.431-447, 2014. https://doi.org/10.1016/j.ejor.2013.09.038

[12] G. Marchet, M. Melacini, S. Perotti and E. Tappia, Analytical model to estimate performances of autonomous vehicle storage and retrieval systems for product totes, International Journal of Production Research, Vol. 50, No. 24, pp. 7134-7148, 2012. https://doi.org/10.1080/00207543.2011.639815

[13] I.F.A. Vis, A comparative analysis of storage and retrieval equipment at a container terminal, International Journal of Production Economics, Vol.103, No.2, pp.680-693, 2006. https://doi.org/10.1016/j.ijpe.2006.01.002

[14] D.J. Fonseca, G. Uppal and T.J. Greene, A knowledge-based system for conveyor equipment selection, Expert Systems with Applications, Vol.26, No.4, pp.615-623, 2004. https://doi.org/10.1016/j.eswa.2003.12.011

[15] M. Wu, Study of three-dimensional simulation platform for autonomous vehicle storage and retrieval system, Automation \& Instrumentation, Vol. 30, No. 8, pp. 1-4, 2015. https://doi.org/10.19557/j.cnki.1001-9944.2015.08.001

[16] N. Zhao, L. Luo, S. Zhang, Z. C. Zhao, Tier captive AVS/RS system retrieval performance analysis based on orthogonal simulation experiment, Computer Integrated Manufacturing Systems, Vol. 22, No. 3, pp. 754-763, 2016. https://doi.org/10.13196/j.cims.2016.03.019

[17] D. Metahri, K. Hachemi, Retrieval-travel-time model for free-fall-flow-rack automated storage and retrieval system, Journal of Industrial Engineering International, Vol. 14, No. 4, pp. 807-820, 2018. http://doi.org/10.1007/s40092-018-0263-9

[18] X. Zou, Y. Wu and Y. Li, Modeling and simulation of centralized and decentralized AS/RS driven by random orders, Computer Integrated Manufacturing Systems, Vol. 23, No.12, pp. 754-763, 2017. https://doi.org/10.13196/j.cims.2017.12.024

[19] S.S. Heragu, X. Cai, A. Krishnamurthy, C.J. Malmborg, Analytical models for analysis of automated warehouse material handling systems, International Journal of Production Research, Vol. 49, No. 22, pp. 6833-6861, 2011. https://doi.org/10.1080/00207543.2010.518994

[20] B.Y. Ekren, Performance evaluation of AVS/RS under various design scenarios: a case study, International Journal of Advanced Manufacturing Technology, Vol. 55, pp. 1253-1261, 2011. http://doi.org/10.1007/s00170-010-3137-x 